\title{
Direction and Stability of Bifurcating Periodic Solutions in a Delay-Induced Ecoepidemiological System
}

\begin{abstract}
N. Bairagi
Centre for Mathematical Biology and Ecology, Department of Mathematics, Jadavpur University, Kolkata 700032, India

Correspondence should be addressed to N. Bairagi, nbairagi@math.jdvu.ac.in

Received 6 May 2011; Accepted 5 July 2011

Academic Editor: Xingfu Zou

Copyright (c) 2011 N. Bairagi. This is an open access article distributed under the Creative Commons Attribution License, which permits unrestricted use, distribution, and reproduction in any medium, provided the original work is properly cited.

A SI-type ecoepidemiological model that incorporates reproduction delay of predator is studied. Considering delay as parameter, we investigate the effect of delay on the stability of the coexisting equilibrium. It is observed that there is stability switches, and Hopf bifurcation occurs when the delay crosses some critical value. By applying the normal form theory and the center manifold theorem, the explicit formulae which determine the stability and direction of the bifurcating periodic solutions are determined. Computer simulations have been carried out to illustrate different analytical findings. Results indicate that the Hopf bifurcation is supercritical and the bifurcating periodic solution is stable for the considered parameter values. It is also observed that the quantitative level of abundance of system populations depends crucially on the delay parameter if the reproduction period of predator exceeds the critical value.
\end{abstract}

\section{Introduction}

Ecoepidemiology is a branch in mathematical biology which considers both the ecological and epidemiological issues simultaneously. After the pioneering work of Anderson and May [1], literature in the field of ecoepidemiology has grown enormously [2-9]. Chattopadhyay and Bairagi [3] studied the following ecoepidemiological model with $m \alpha=\theta$ :

$$
\begin{gathered}
\frac{d S}{d t}=r S\left(1-\frac{S+I}{K}\right)-\lambda I S, \\
\frac{d I}{d t}=\lambda I S-\frac{m I P}{a+I}-\mu I, \\
\frac{d P}{d t}=\frac{m \alpha I P}{a+I}-d P .
\end{gathered}
$$


In this model, $S, I$, and $P$ represent the densities of susceptible prey, infected prey, and the predator populations, respectively. Both susceptible and infected preys contribute to the carrying capacity $(K)$, but only susceptible prey can reproduce at the intrinsic growth rate $r$. Disease spreads horizontally from infected to susceptible prey at a rate $\lambda$ following the law of mass action. Predator preys on infected prey only and predation process follows Holling Type II [10] response function with search rate $m$ and half-saturation constant $a$. Here, $\alpha$ is the conversion efficiency of the predator defining the increase in predator's number per unit prey consumption. $\mu\left(=\mu_{1}+\mu_{2}\right)$ represents the total death rate of infected prey where $\mu_{1}$ is the natural death rate and $\mu_{2}$ is the virulence of the disease. Predators consume both the susceptible and infected preys; however, the predation rate on infected prey may be very high (31 times) compare to that on susceptible prey [11]. Based on the experimental observation [11], it is assumed that predator consumes infected prey only. Predators may have to pay a cost in terms of extra mortality in the tradeoff between the easier predation and the parasitized prey acquisition, but the benefit is assumed to be greater than the cost $[12,13]$. So it is assumed that consumption of infected prey contributes positive growth to the predator population. $d\left(=d_{1}+d_{2}\right)$ is the total death rate of predator where $d_{1}$ is the natural death rate and $d_{2}$ is the cost due to parasitized prey acquisition. All parameters are assumed to be positive.

Reproduction of predator after consuming the prey is not instantaneous, but mediated by some time lag. Chattopadhyay and Bairagi [3] did not consider this reproduction delay, defined by the time required for the reproduction of predator after consuming the prey, in their model system. It is well recognized that introduction of reproduction delay makes the model biologically more realistic. If $\tau(>0)$ is the time required for the reproduction, the model (1.1) can be written as

$$
\begin{gathered}
\frac{d S}{d t}=r S\left(1-\frac{S+I}{K}\right)-\lambda I S, \\
\frac{d I}{d t}=\lambda I S-\frac{m I P}{a+I}-\mu I, \\
\frac{d P}{d t}=\frac{m \alpha I(t-\tau) P(t-\tau)}{a+I(t-\tau)}-d P .
\end{gathered}
$$

We study the delay-induced system (1.2) with the following initial conditions:

$$
S(\theta)=\psi_{1}(\theta) \geq 0, \quad I(\theta)=\psi_{2}(\theta) \geq 0, \quad P(\theta)=\psi_{3}(\theta) \geq 0, \quad \theta \in(-\tau, 0] .
$$

Hopf bifurcation and its stability in a delay-induced predator-prey system have been studied by many researchers [14-19]. In this paper, we study the effect of reproduction delay on an ecoepidemiological system where predator-prey interaction follows Holling Type II response function, and find the direction and stability of the bifurcating periodic solutions, if any.

The organization of the paper is as follows. Section 2 deals with the linear stability analysis of the model system. In Section 3, direction and stability of Hopf bifurcation are presented. Numerical results to illustrate the analytical findings are presented in Section 4 and, finally, a summary is presented in Section 5. 


\section{Stability Analysis and Hopf Bifurcation}

In epidemiology, the basic reproductive ratio $R_{0}$, the number of new cases acquired directly from a single infected prey when introduced into a population of susceptible, plays a significant role in the spread of the disease. In particular, if $R_{0}<1$, the disease dies out, but if $R_{0}>1$, it remains endemic in the host population [20]. For the system (1.2), the basic reproductive ratio is given by $R_{0}=\lambda K / \mu$. In ecology, on the other hand, stress is given on the stability of coexisting equilibrium point. We, therefore, concentrate on the study of the stability of the coexisting or endemic equilibrium point of the system (1.2). The ecoepidemiological system (1.2) has a unique interior equilibrium point $E^{*}\left(S^{*}, I^{*}, P^{*}\right)$, where $S^{*}=K-(a d(r+\lambda K) / r(m \alpha-d)), I^{*}=a d /(m \alpha-d)$, and $P^{*}=(1 / m)\left(a+I^{*}\right)\left(\lambda S^{*}-\mu\right)$. Note that $I^{*}$ exists if $m>d / \alpha, S^{*}$ exists if $m>d / \alpha+(a d(r+\lambda K) / r K \alpha)$ and $P^{*}$ exists if $m>(d / \alpha)+(a d \lambda(r+\lambda K) / r \alpha(\lambda K-\mu))=(d / \alpha)+(a d(r+\lambda K) / r K \alpha-(r \alpha \mu / \lambda))$ with $\lambda>\mu / K$. Thus, the conditions for coexisting equilibrium point $E^{*}$ are

(i) $\lambda>\mu / K$, that is, $R_{0}>1$,

(ii) $m>(d / \alpha)+(a d \lambda(r+\lambda K) / r \alpha(\lambda K-\mu))$.

Let $x(t)=S(t)-S^{*}, y(t)=I(t)-I^{*}$, and $z(t)=P(t)-P^{*}$ be the perturbed variables. Then, the system (1.2) can be expressed in the matrix form after linearization as follows:

$$
\frac{d}{d t}\left(\begin{array}{l}
x(t) \\
y(t) \\
z(t)
\end{array}\right)=A_{1}{ }^{\prime}\left(\begin{array}{l}
x(t) \\
y(t) \\
z(t)
\end{array}\right)+A_{2}{ }^{\prime}\left(\begin{array}{l}
x(t-\tau) \\
y(t-\tau) \\
z(t-\tau)
\end{array}\right),
$$

where

$$
\begin{gathered}
A_{1}{ }^{\prime}=\left(\begin{array}{ccc}
-\frac{r S^{*}}{K} & -\left(\lambda+\frac{r}{K}\right) S^{*} & 0 \\
\lambda I^{*} & \frac{m I^{*} P^{*}}{\left(a+I^{*}\right)^{2}} & -\frac{d}{\alpha} \\
0 & 0 & -d
\end{array}\right), \\
A_{2}{ }^{\prime}=\left(\begin{array}{ccc}
0 & 0 & 0 \\
0 & 0 & 0 \\
0 & \frac{\alpha a m P^{*}}{\left(a+I^{*}\right)^{2}} & d
\end{array}\right) .
\end{gathered}
$$

The characteristic equation of the system (2.1) is given by

$$
\left|A_{1}{ }^{\prime}+A_{2}{ }^{\prime} e^{-\xi \tau}-\xi I\right|=0
$$

that is,

$$
\Phi(\xi, \tau)=\xi^{3}+\left[A+B e^{-\xi \tau}\right] \xi^{2}+\left[C+D e^{-\xi \tau}\right] \xi+E+F e^{-\xi \tau}=0
$$


where

$$
\begin{gathered}
A=\frac{r}{K} S^{*}+d-\frac{m I^{*} P^{*}}{\left(a+I^{*}\right)^{2}}, \\
B=-d, \\
C=-\frac{m d I^{*} P^{*}}{\left(a+I^{*}\right)^{2}}+\frac{d r S^{*}}{K}-\frac{r m S^{*} I^{*} P^{*}}{K\left(a+I^{*}\right)^{2}}+\left(\frac{r}{K}+\lambda\right) \lambda S^{*} I^{*}, \\
D=\frac{m d I^{*} P^{*}}{\left(a+I^{*}\right)^{2}}-\frac{d r S^{*}}{K}+\frac{a d m P^{*}}{\left(a+I^{*}\right)^{2}}, \\
E=-\frac{d r m S^{*} I^{*} P^{*}}{K\left(a+I^{*}\right)^{2}}+\left(\frac{r}{K}+\lambda\right) \curlywedge d S^{*} I^{*}, \\
F=\frac{d r m S^{*} I^{*} P^{*}}{K\left(a+I^{*}\right)^{2}}+\frac{a d r m S^{*} P^{*}}{K\left(a+I^{*}\right)^{2}}-\left(\frac{r}{K}+\lambda\right) \lambda d S^{*} I^{*} .
\end{gathered}
$$

Equation (2.4) can be written as

$$
\Phi(\xi, \tau)=\xi^{3}+m_{2} \xi^{2}+m_{1} \xi+m_{0}+\left(n_{2} \xi^{2}+n_{1} \xi+n_{0}\right) e^{-\xi \tau}=0,
$$

where

$$
\begin{array}{cc}
m_{2}=A, & n_{2}=B, \\
m_{1}=C, & n_{1}=D, \\
m_{0}=E, & n_{0}=F \\
\Sigma n_{i}^{2} \neq 0, & (i=0,1,2) .
\end{array}
$$

For $\tau=0$, (2.5) becomes

$$
\begin{aligned}
\Phi(\xi, 0) & =\xi^{3}+\left(m_{2}+n_{2}\right) \xi^{2}+\left(m_{1}+n_{1}\right) \xi+\left(m_{0}+n_{0}\right) \\
& =\xi^{3}+X \xi^{2}+Y \xi+Z=0 .
\end{aligned}
$$

Here

$$
X=m_{2}+n_{2}=\frac{r S^{*}}{K}-\frac{m P^{*} I^{*}}{\left(a+I^{*}\right)^{2}}=\left(\frac{r}{K}-\frac{d \lambda}{m \alpha}\right) S^{*}+\frac{d \mu}{m \alpha} .
$$

Thus, $X>0$ if $m>d \lambda K / r \alpha$. After some algebraic manipulation, $Y$ can be written as

$$
Y=m_{1}+n_{1}=\frac{r m a S^{*} P^{*}}{K\left(a+I^{*}\right)^{2}}+\frac{m a d P^{*}}{\left(a+I^{*}\right)^{2}}+S^{*}\left\{\frac{r \mu}{K}+\lambda I^{*}\left(\lambda+\frac{r}{K}\right)-\frac{r \lambda S^{*}}{K}\right\} .
$$


So the sufficient condition for $Y$ to be positive is

$$
\frac{r \mu}{K}+\lambda I^{*}\left(\lambda+\frac{r}{K}\right)>\frac{r \lambda S^{*}}{K} \quad \text { or } \quad m<\frac{d}{\alpha}+\frac{2 \lambda a d(r+\lambda K)}{r \alpha(\lambda K-\mu)} .
$$

Note that $Z=m_{0}+n_{0}=\operatorname{adrm} S^{*} P^{*} / K\left(a+I^{*}\right)^{2}$ is always positive. One can write,

$$
\begin{aligned}
X Y-Z= & {\left[\left(\frac{r}{K}-\frac{d \lambda}{m \alpha}\right) S^{*}\right]\left[\left\{\frac{r m a S^{*} P^{*}}{K\left(a+I^{*}\right)^{2}}+\frac{m a d P^{*}}{\left(a+I^{*}\right)^{2}}\right\}+\left\{\frac{r \mu S^{*}}{K}+\lambda S^{*} I^{*}\left(\lambda+\frac{r}{K}\right)-\frac{r \lambda S^{* 2}}{K}\right\}\right] } \\
& +\frac{d \mu}{m \alpha}\left[\frac{m a d P^{*}}{\left(a+I^{*}\right)^{2}}\right]+\frac{d \mu}{m \alpha}\left[\frac{r \mu S^{*}}{K}+\lambda S^{*} I^{*}\left(\lambda+\frac{r}{K}\right)-\frac{\lambda r S^{* 2}}{K}\right] \\
& +\frac{d \mu}{m \alpha} \frac{r m a S^{*} P^{*}}{K\left(a+I^{*}\right)^{2}}-\frac{r m a d S^{*} P^{*}}{K\left(a+I^{*}\right)^{2}} .
\end{aligned}
$$

Since all the terms in the third bracket are positive, so the sufficient condition for the positivity of $(X Y-E)$ is

$$
\frac{d \mu}{m \alpha} \frac{\operatorname{rmaS}^{*} P^{*}}{K\left(a+I^{*}\right)^{2}}>\frac{\operatorname{rmad} S^{*} P^{*}}{K\left(a+I^{*}\right)^{2}} \quad \text { or } \quad m<\frac{\mu}{\alpha}
$$

Hence, by Routh-Hurwitz criterion and using existence conditions, we state the following theorem for the stability of the interior equilibrium $E^{*}$ of the system (1.2) for $\tau=0$.

Theorem 2.1. If

(i) $R_{0}>1$ or $\lambda>\mu / K$,

(ii) $\underline{m}<m<\bar{m}$, where $\underline{m}=\max [d \lambda K / r \alpha,(d / \alpha)+(\operatorname{ad\lambda }(r+\lambda K) / r \alpha(\lambda K-\mu))]$ and $\bar{m}=$ $\min [\mu / \alpha,(d / \alpha)+(2 \lambda a d(r+\lambda K) / r \alpha(\lambda K-\mu))]$,

then the system (1.2) is locally asymptotically stable without delay around the positive interior equilibrium $E^{*}$.

We now reproduce some definitions given by [21, 22].

Definition 2.2. The equilibrium $E^{*}$ is called asymptotically stable if there exists a $\delta>0$ such that

$$
\sup _{-\tau \leq \theta \leq 0}\left[\left|\psi_{1}(\theta)-S^{*}\right|,\left|\psi_{2}(\theta)-I^{*}\right|,\left|\psi_{3}(\theta)-P^{*}\right|\right]<\delta
$$

implies that

$$
\lim _{t \rightarrow \infty}(S(t), I(t), P(t))=\left(S^{*}, I^{*}, P^{*}\right)
$$

where $(S(t), I(t), P(t))$ is the solution of the system (1.2) which satisfies the condition (1.3). 
Definition 2.3. The equilibrium $E^{*}$ is called absolutely stable if it is asymptotically stable for all delays $\tau \geq 0$ and conditionally stable if it is stable for $\tau$ in some finite interval.

Note that the system (1.2) will be stable around the equilibrium $E^{*}$ if all the roots of the corresponding characteristic equation (2.5) have negative real parts. But (2.5) is a transcendental equation and has infinite number of roots. It is difficult to determine the sign of these infinite number of roots. Therefore, we first study the distribution of roots of the cubic exponential polynomial equation (2.5).

We know that $i \omega(\omega>0)$ is a root of (2.5) if and only if $\omega$ satisfies

$$
-i \omega^{3}-\omega^{2} m_{2}+m_{1} i \omega+m_{0}+\left(-n_{2} \omega^{2}+n_{1} i \omega+n_{0}\right)(\cos \omega \tau-i \sin \omega \tau)=0 .
$$

Separating real and imaginary parts, we get

$$
\begin{gathered}
m_{2} \omega^{2}-m_{0}=-n_{2} \omega^{2} \cos \omega \tau+n_{1} \omega \sin \omega \tau+n_{0} \cos \omega \tau, \\
\omega^{3}-m_{1} \omega=n_{2} \omega^{2} \sin \omega \tau+n_{1} \omega \cos \omega \tau-n_{0} \sin \omega \tau .
\end{gathered}
$$

This two equations give the positive values of $\tau$ and $\omega$ for which (2.5) can have purely imaginary roots.

Squaring and adding, we obtain

$$
\omega^{6}+p \omega^{4}+q \omega^{2}+s=0
$$

where

$$
\begin{gathered}
p=m_{2}^{2}-2 m_{1}-n_{2}^{2}, \\
q=m_{1}^{2}-2 m_{0} m_{2}+2 n_{0} n_{2}-n_{1}^{2}, \\
s=\left(m_{0}^{2}-n_{0}^{2}\right) .
\end{gathered}
$$

If we assume $h=\omega^{2}$, then (2.16) reduces to

$$
h^{3}+p h^{2}+q h+s=0 .
$$

Denote

$$
g(h)=h^{3}+p h^{2}+q h+s
$$

Note that $g(0)=s$ and $\lim _{h \rightarrow+\infty} g(h)=+\infty$. Thus, if $s<0$, then (2.18) has at least one positive root.

From (2.18), we have

$$
g^{\prime}(h)=3 h^{2}+2 p h+q
$$


Clearly, if $\Delta=p^{2}-3 q \leq 0$, then the function $g(h)$ is monotonically increasing in $h \in[0, \infty)$. Thus, for $s \geq 0$ and $\Delta \leq 0,(2.18)$ has no positive roots for $h \in[0, \infty)$. On the other hand, when $s \geq 0$ and $\Delta<0$, the equation

$$
3 h^{2}+2 p h+q=0
$$

has two real roots

$$
h_{1}^{*}=\frac{-p+\sqrt{\Delta}}{3}, \quad h_{2}^{*}=\frac{-p-\sqrt{\Delta}}{3} .
$$

Obviously, $g^{\prime \prime}\left(h_{1}^{*}\right)=2 \sqrt{\Delta}>0$ and $g^{\prime \prime}\left(h_{2}^{*}\right)=-2 \sqrt{\Delta}<0$. It follows that $h_{1}^{*}$ and $h_{2}^{*}$ are the local minimum and the local maximum, respectively. Hence we have the following lemma.

Lemma 2.4. Suppose that $s \geq 0$ and $\Delta>0$. Then (2.17) has positive roots if and only if $h_{1}^{*}>$ $0, g\left(h_{1}^{*}\right) \leq 0$.

Proof. Noticing that $s \geq 0, h_{1}^{*}$ is the local minimum of $g(h)$ and $\lim _{h \rightarrow+\infty} g(h)=+\infty$, we immediately know that the sufficiency is true. So we have to prove now the necessity. In contrary, we suppose that either $h_{1}^{*} \leq 0$ or $h_{1}^{*}>0$ and $g\left(h_{1}^{*}\right)>0$. Since $g(h)$ is increasing for $h \geq h_{1}^{*}$ and $g(0)=s \geq 0$, it follows that $g(h)$ has no positive real roots for $h_{1}^{*} \leq 0$ and $g\left(h_{1}^{*}\right)>0$. If $h_{1}^{*}>0$ and $g\left(h_{1}^{*}\right)>0$, since $h_{2}^{*}$ is the local maximum value, it follows that $g\left(h_{1}^{*}\right)<g\left(h_{2}^{*}\right)$. Thus, $g(z)$ cannot have any positive real roots when $h_{1}^{*}>0$ and $g\left(h_{1}^{*}\right)>0$. This completes the proof.

Summarizing the above discussions, we obtain the following.

Lemma 2.5. One has the following results on the distribution of roots of (2.17).

(i) If $s<0$, then (2.17) has at least one positive root;

(ii) if $s \geq 0$, and $\Delta=p^{2}-3 q \leq 0$, then (2.17) has no positive root;

(iii) if $s \geq 0$, and $\Delta=p^{2}-3 q>0$, then (2.17) has positive roots if and only if $h_{1}^{*}=(-p+$ $\sqrt{\Delta}) / 3>0$ and $g\left(h_{1}^{*}\right) \leq 0$, where $g(z)=h^{3}+p h^{2}+q h+s$.

Suppose that (2.17) has positive roots. Without loss of generality, we assume that it has three positive roots, defined by $h_{1}, h_{2}$, and $h_{3}$, respectively. Then, (2.16) has three positive roots $\omega_{1}=\sqrt{h_{1}}, \omega_{2}=\sqrt{h_{2}}$, and $\omega_{3}=\sqrt{h_{3}}$.

From (2.15), we have

$$
\cos \omega_{k} \tau=\frac{\left(n_{1}-n_{2} m_{2}\right) \omega^{4}+\left(n_{2} m_{0}+n_{0} m_{2}-n_{1} m_{1}\right) \omega^{2}-n_{0} m_{0}}{\left(n_{2} \omega^{2}-n_{0}\right)^{2}+n_{1}^{2} w^{2}}, \quad k=1,2,3 .
$$

Thus, if we denote

$$
\tau_{k}^{j}=\frac{1}{\omega_{k}}\left\{\arccos \left[\frac{\left(n_{1}-n_{2} m_{2}\right) \omega_{k}^{4}+\left(n_{2} m_{0}+n_{0} m_{2}-n_{1} m_{1}\right) \omega_{k}^{2}-n_{0} m_{0}}{\left(n_{2} \omega_{k}^{2}-n_{0}\right)^{2}+n_{1}^{2} w_{k}^{2}}\right]+2 j \pi\right\}
$$


where $k=1,2,3 ; j=0,1,2, \ldots$, then $\pm i \omega_{k}$ is a pair of purely imaginary roots of (2.5). Define

$$
\tau_{0}=\tau_{k_{0}}^{(0)}=\min _{k \in\{1,2,3\}}\left\{\tau_{k}^{(0)}\right\}, \quad \omega_{0}=\omega_{k_{0}} .
$$

We reproduce the following result due to Ruan and Wei [23] to analyze (2.5).

Lemma 2.6. Consider the exponential polynomial

$$
\begin{aligned}
P\left(\xi, e^{-\xi \tau_{1}}, \ldots, e^{-\xi \tau_{m}}\right)= & \xi^{n}+p_{1}^{(0)} \xi^{n-1}+\cdots+p_{n-1}^{(0)} \xi+p_{n}^{(0)} \\
& +\left[p_{1}^{(1)} \xi^{n-1}+\cdots+p_{n-1}^{(1)} \xi+p_{n}^{(1)}\right] e^{-\xi \tau_{1}} \\
& +\cdots+\left[p_{1}^{(m)} \xi^{n-1}+\cdots+p_{n-1}^{(m)} \xi+p_{n}^{(m)}\right] e^{-\xi \tau_{m}},
\end{aligned}
$$

where $\tau_{i} \geq 0(i=1,2, \ldots, m)$ and $p_{j}^{(i)},(i=0,1,2, \ldots, m ; j=1,2, \ldots, n)$ are constants. As $\left(\tau_{1}, \tau_{2}, \ldots, \tau_{m}\right)$ vary, the sum of the order of zeros of $P\left(\xi, e^{-\xi \tau_{1}}, \ldots, e^{-\xi \tau_{m}}\right)$ on the open right half hand can change only if a zero appears on or crosses the imaginary axis.

Using Lemmas 2.5 and 2.6, we can easily obtain the following results on the distribution of roots of the transcendental (2.5).

Lemma 2.7. For the third degree exponential polynomial equation (2.5), one has

(i) if $s \geq 0$, and $\Delta=p^{2}-3 q \leq 0$, then all roots with positive real parts of (2.5) have the same sum as those of the polynomial equation (2.6) for all $\tau \geq 0$,

(ii) if either $s<0$ or $s \geq 0, \Delta=p^{2}-3 q>0, h_{1}^{*}=(-p+\sqrt{\Delta}) / 3>0$ and $g\left(h_{1}^{*}\right) \leq 0$, then all roots with positive real parts of (2.5) have the same sum as those of the polynomial equation (2.6) for all $\tau \in\left[0, \tau_{0}\right)$.

Let

$$
\xi(\tau)=\eta(\tau)+i \omega(\tau)
$$

where $\eta$ and $\omega$ are real, be the roots of (2.5) near $\tau=\tau_{k}^{(j)}$ satisfying

$$
\eta\left(\tau_{k}^{(j)}\right)=0, \quad \omega\left(\tau_{k}^{(j)}\right)=\omega_{k}
$$

Then the following transversality condition holds.

Lemma 2.8. Suppose that $h_{k}=\omega_{k}^{2}$ and $g^{\prime}\left(h_{k}\right) \neq 0$, where $g(h)$ is defined by (2.18). Then,

$$
\frac{d}{d \tau}\left[\operatorname{Re}\left\{\xi\left(\tau_{k}^{(j)}\right)\right\}\right] \neq 0,
$$

and the sign of $(d / d \tau)\left[\operatorname{Re}\left\{\xi\left(\tau_{k}^{(j)}\right)\right\}\right]$ is consistent with that of $g^{\prime}\left(h_{k}\right)$. 
Proof. Differentiating (2.5) with respect to $\tau$, we obtain

$$
\left[\left(3 \xi^{2}+2 m_{2} \xi+m_{1}\right)+e^{-\xi \tau}\left\{2 n_{2} \xi+n_{1}-\tau\left(n_{2} \xi^{2}+n_{1} \xi+n_{0}\right)\right\}\right] \frac{d \xi}{d \tau}=\xi\left(n_{2} \xi^{2}+n_{1} \xi+n_{0}\right) e^{-\xi \tau}
$$

This gives

$$
\left(\frac{d \xi}{d \tau}\right)^{-1}=\frac{\left(3 \xi^{2}+2 m_{2} \xi+m_{1}\right) e^{\xi \tau}}{\xi\left(n_{2} \xi^{2}+n_{1} \xi+n_{0}\right)}+\frac{2 n_{2} \xi+n_{1}}{\xi\left(n_{2} \xi^{2}+n_{1} \xi+n_{0}\right)}-\frac{\tau}{\xi}
$$

It follows from (2.15) that

$$
\begin{gathered}
{\left[\xi\left(n_{2} \xi^{2}+n_{1} \xi+n_{0}\right)\right]_{\tau=\tau_{k}^{(j)}}=-n_{1} \omega_{k}^{2}+i\left(n_{0} \omega_{k}-n_{2} \omega_{k}^{3}\right)} \\
{\left[\left(3 \xi^{2}+2 m_{2} \xi+m_{1}\right) e^{\xi \tau}\right]_{\tau=\tau_{k}^{(j)}}=\left[\left(m_{1}-3 \omega_{k}^{2}\right) \cos \omega_{k} \tau_{k}^{(j)}-2 m_{2} \omega_{k} \sin \omega_{k} \tau_{k}^{(j)}\right]} \\
+i\left[\left(m_{1}-3 \omega_{k}^{2}\right) \sin \omega_{k} \tau_{k}^{(j)}+2 m_{2} \omega_{k} \cos \omega_{k} \tau_{k}^{(j)}\right] \\
{\left[2 n_{2} \xi+n_{1}\right]_{\tau=\tau_{k}^{(j)}}=n_{1}+i 2 n_{2} \omega_{k} .}
\end{gathered}
$$

Using (2.31) in (2.30), we get

$$
\begin{aligned}
\frac{d}{d \tau}[\operatorname{Re}\{\xi(\tau)\}]_{\tau=\tau_{k}^{(j)}}^{-1}= & \operatorname{Re}\left\{\frac{\left(3 \xi^{2}+2 m_{2} \xi+m_{1}\right) e^{\xi \tau}}{\xi\left(n_{2} \xi^{2}+n_{1} \xi+n_{0}\right)}\right\}_{\tau=\tau_{k}^{(j)}} \\
+ & \operatorname{Re}\left\{\frac{2 n_{2} \xi+n_{1}}{\xi\left(n_{2} \xi^{2}+n_{1} \xi+n_{0}\right)}\right\}_{\tau=\tau_{k}^{(j)}}-\operatorname{Re}\left\{\frac{\tau}{\xi}\right\}_{\tau=\tau_{k}^{(j)}} \\
=\frac{1}{\Lambda}\left[\omega_{k}\left(m_{1}-3 \omega_{k}^{2}\right)\left\{-n_{1} \omega_{k} \cos \omega_{k} \tau_{k}^{(j)}+\left(n_{0}-n_{2} \omega_{k}^{2}\right) \sin \omega_{k} \tau_{k}^{(j)}\right\}\right. & +2 m_{2} \omega_{k}^{2}\left\{n_{1} \omega_{k} \sin \omega_{k} \tau_{k}^{(j)}+\left(n_{0}-n_{2} \omega_{k}^{2}\right) \cos \omega_{k} \tau_{k}^{(j)}\right\} \\
& \left.\quad-n_{1}^{2} \omega_{k}^{2}+2 n_{2} \omega_{k}^{2}\left(n_{0}-n_{2} \omega_{k}^{2}\right)\right] \\
= & \frac{1}{\Lambda}\left[\omega_{k}\left(m_{1}-3 \omega_{k}^{2}\right)\left(m_{1} \omega_{k}-\omega_{k}^{3}\right)+2 m_{2} \omega_{k}^{2}\left(m_{2} \omega_{k}^{2}-m_{0}\right)\right. \\
& \left.\quad-n_{1}^{2} \omega_{k}^{2}+2 n_{2} \omega_{k}^{2}\left(n_{0}-n_{2} \omega_{k}^{2}\right)\right]
\end{aligned}
$$




$$
\begin{aligned}
& =\frac{1}{\Lambda}\left[3 \omega_{k}^{6}+2\left(m_{2}^{2}-2 m_{1}-n_{2}^{2}\right) \omega_{k}^{4}+\left(m_{1}^{2}-2 m_{0} m_{2}-n_{1}^{2}+2 n_{0} n_{2}\right) \omega_{k}^{2}\right] \\
& =\frac{1}{\Lambda}\left[3 \omega_{k}^{6}+2 p \omega_{k}^{4}+q \omega_{k}^{2}\right] \\
& =\frac{1}{\Lambda} h_{k} g^{\prime}\left(h_{k}\right),
\end{aligned}
$$

where $\Lambda=n_{1}^{2} \omega_{k}^{4}+\left(n_{0}-n_{2} \omega_{k}^{2}\right)^{2}(>0)$. Thus, we have

$$
\operatorname{sign}\left\{\frac{d}{d \tau} \operatorname{Re} \xi(\tau)\right\}_{\tau=\tau_{k}^{(j)}}=\operatorname{sign}\left\{\frac{d}{d \tau} \operatorname{Re} \xi(\tau)\right\}_{\tau=\tau_{k}^{(j)}}^{-1}=\operatorname{sign}\left\{\frac{h_{k}}{\Lambda} g^{\prime}\left(h_{k}\right)\right\} \neq 0 .
$$

Since $\Lambda, h_{k}$ are positive, we conclude that the sign of $\{(d / d \tau) \operatorname{Re} \xi(\tau)\}_{\tau=\tau_{k}^{(j)}}$ is determined by that of $g^{\prime}\left(h_{k}\right)$. This proves the lemma.

From $(2.5)^{\prime}$ and $(2.16)^{\prime}$, we have

$$
\begin{gathered}
p=m_{2}^{2}-2 m_{1}-n_{2}^{2}=A^{2}-2 C^{2}-B^{2}, \\
q=m_{1}^{2}-2 m_{0} m_{2}+2 n_{0} n_{2}-n_{1}^{2}=C^{2}-2 A E+2 B F-D^{2}, \\
s=m_{0}^{2}-n_{0}^{2}=E^{2}-F^{2} .
\end{gathered}
$$

Thus, from Lemmas 2.7 and 2.8, we have the following theorem.

Theorem 2.9. Let $m_{i}, n_{i}(i=0,1,2) ; p, q, s$ and $\tau^{j}$ are defined $b\left((2.5)^{\prime}\right)$, (2.34), and (2.23), respectively. Suppose that conditions of Theorem (2.1) hold. Then the following results hold.

(i) When $s \geq 0$, and $\Delta=p^{2}-3 q \leq 0$, then all roots of (2.5) have negative real parts for all $\tau \geq 0$ and the equilibrium $E^{*}$ of the system (1.2) is absolutely stable for all $\tau \geq 0$.

(ii) If either $s<0$ or $s \geq 0, \Delta=p^{2}-3 q>0, h_{1}^{*}=(-p+\sqrt{\Delta}) / 3>0$ and $g\left(h_{1}^{*}\right) \leq 0$ hold, then $g(h)$ has at least one positive root $h_{k}$ and all roots of (2.5) have negative real parts for all $\tau \in\left[0, \tau_{k}^{(0)}\right)$, then the equilibrium $E^{*}$ of the system (1.2) is conditionally stable for $\tau \in\left[0, \tau_{k}^{(0)}\right)$.

(iii) If all the conditions as stated in (ii) and $g^{\prime}\left(h_{k}\right) \neq 0$ hold, then the system (1.2) undergoes a Hopf bifurcation at $E^{*}$ when $\tau=\tau_{k}^{(j)},(j=0,1,2, \ldots)$.

\section{Direction and Stability of the Hopf Bifurcation}

In the previous section, we obtained some conditions under which system (1.2) undergoes Hopf bifurcation at $\tau=\tau^{(j)}(j=0,1,2, \ldots)$. In this section, we assume that the system (1.2) undergoes Hopf bifurcation at $E^{*}$ when $\tau=\tau^{(j)}$, that is, a family of periodic solutions bifurcate from the positive equilibrium point $E^{*}$ at the critical value $\tau=\tau^{(j)}(j=0,1,2, \ldots)$. We will use 
the normal form theory and center manifold presented by Hassard et al. [24] to determine the direction of Hopf bifurcation, that is, to ensure whether the bifurcating branch of periodic solution exists locally for $\tau>\tau^{(j)}$ or $\tau<\tau^{(j)}$, and determine the properties of bifurcating periodic solutions, for example, stability on the center manifold and period. Throughout this section, we always assume that system (1.2) undergoes Hopf bifurcation at the positive equilibrium $E^{*}\left(S^{*}, I^{*}, P^{*}\right)$ for $\tau=\tau^{(j)}$ and then $\pm i \omega_{k}$ is corresponding purely imaginary roots of the characteristic equation.

Let $x_{1}=S-S^{*}, x_{2}=I-I^{*}, x_{3}=P-P^{*}, \bar{x}_{i}(t)=x_{i}(\tau t), \tau=\tau^{(j)}+\nu$, where $\tau^{(j)}$ is defined by (2.23) and $v \in R$. Dropping the bars for simplification of notations, system (1.2) can be written as functional differential equation (FDE) in $C=C\left([-1,0], R^{3}\right)$ as

$$
\dot{x}(t)=L_{v}\left(x_{t}\right)+f\left(v, x_{t}\right),
$$

where $x(t)=\left(x_{1}, x_{2}, x_{3}\right)^{T} \in R^{3}$, and $L_{v}: C \rightarrow R, f: R \times C \rightarrow R$ are given, respectively, by

$$
\begin{array}{r}
L_{v}(\phi)=\left(\tau^{(j)}+v\right)\left(\begin{array}{ccc}
-\frac{r S^{*}}{K} & -\left(\frac{r}{K}+\lambda\right) S^{*} & 0 \\
\lambda I^{*} & \frac{m I^{*} P^{*}}{\left(a+I^{*}\right)^{2}} & -\frac{d}{\alpha} \\
0 & 0 & -d
\end{array}\right)\left(\begin{array}{l}
\phi_{1}(0) \\
\phi_{2}(0) \\
\phi_{3}(0)
\end{array}\right) \\
+\left(\tau^{(j)}+v\right)\left(\begin{array}{ccc}
0 & 0 & 0 \\
0 & 0 & 0 \\
0 & \frac{\alpha a m P^{*}}{\left(a+I^{*}\right)^{2}} & d
\end{array}\right)\left(\begin{array}{l}
\phi_{1}(-1) \\
\phi_{2}(-1) \\
\phi_{3}(-1)
\end{array}\right), \\
f(v, \phi)=\left(\tau^{(j)}+v\right)\left(\begin{array}{c}
-\frac{r}{K}\left(\phi_{1}^{2}(0)+\phi_{1}(0) \phi_{2}(0)\right)-\lambda \phi_{1}(0) \phi_{2}(0) \\
\lambda \phi_{1}(0) \phi_{2}(0)-\frac{m \phi_{2}(0) \phi_{3}(0)}{a+\phi_{2}(0)} \\
\frac{\alpha m \phi_{2}(-1) \phi_{3}(-1)}{a+\phi_{2}(-1)}
\end{array}\right) .
\end{array}
$$

By the Riesz representation theorem, there exists a $(3 \times 3)$ matrix, $\eta(\theta, v)(-1 \leq \theta \leq 0)$ whose elements are bounded variation functions such that

$$
L_{\nu} \phi=\int_{-1}^{0} d \eta(\theta, v) \phi(\theta), \quad \text { for } \phi \in C
$$


In fact, we can choose

$$
\eta(\theta, v)=\left(\tau^{(j)}+v\right)\left(\begin{array}{ccc}
-\frac{r S^{*}}{K} & -\left(\frac{r}{K}+\lambda\right) S^{*} & 0 \\
\lambda I^{*} & \frac{m I^{*} P^{*}}{\left(a+I^{*}\right)^{2}} & -\frac{d}{\alpha} \\
0 & 0 & -d
\end{array}\right) \delta(\theta)-\left(\tau^{(j)}+v\right)\left(\begin{array}{ccc}
0 & 0 & 0 \\
0 & 0 & 0 \\
0 & \frac{\alpha a m P^{*}}{\left(a+I^{*}\right)^{2}} & d
\end{array}\right) \delta(\theta+1)
$$

where $\delta$ is the Dirac delta function defined by

$$
\delta(\theta)= \begin{cases}0, & \theta \neq 0 \\ 1, & \theta=0\end{cases}
$$

For $\phi \in C^{1}\left([-1,0], R^{3}\right)$, define the operator $A(v)$ as

$$
\begin{gathered}
A(v) \phi(\theta)= \begin{cases}\frac{d \phi(\theta)}{d \theta}, & \theta \in[-1,0), \\
\int_{-1}^{0} d \eta(v, s) \phi(s), & \theta=0,\end{cases} \\
R(v) \phi(\theta)= \begin{cases}0, & \theta \in[-1,0) \\
f(v, \phi), & \theta=0 .\end{cases}
\end{gathered}
$$

Then system (3.1) is equivalent to

$$
\dot{x}(t)=A(v) x_{t}+R(v) x_{t},
$$

where $x_{t}(\theta)=x(t+\theta)$ for $\theta \in[-1,0]$.

For $\psi \in C^{1}\left([0,1],\left(R^{3}\right)^{*}\right)$, define

$$
A^{*} \psi(s)= \begin{cases}-\frac{d \psi(s)}{d s}, & s \in(0,1] \\ \int_{-1}^{0} d \eta^{T}(t, 0) \psi(-t), & s=0\end{cases}
$$

and a bilinear inner product

$$
\langle\psi(s), \phi(\theta)\rangle=\bar{\psi}(0) \phi(0)-\int_{-1}^{0} \int_{\xi=0}^{\theta} \bar{\psi}(\xi-\theta) d \eta(\theta) \phi(\xi) d \xi,
$$


where $\eta(\theta)=\eta(\theta, 0)$. Then $A(0)$ and $A^{*}$ are adjoint operators. By Theorem 2.9, we know that $\pm i \tau^{(j)} \omega_{0}$ are eigenvalues of $A(0)$. Thus, they are also eigenvalues of $A^{*}$. We first need to compute the eigenvalues of $A(0)$ and $A^{*}$ corresponding to $+i \tau^{(j)} \omega_{0}$ and $-i \tau^{(j)} \omega_{0}$, respectively.

Suppose that $q(\theta)=(1, \beta, \gamma)^{T} e^{i \theta \omega_{0} \tau^{(j)}}$ is the eigenvector of $A(0)$ corresponding to $i \tau^{(j)} \omega_{0}$. Then $A(0) q(\theta)=i \omega_{0} \tau^{(j)} q(\theta)$. It follows from the definition of $A(0)$ and (3.2), (3.4), and (3.5) that

$$
\tau^{(j)}\left(\begin{array}{ccc}
i \omega_{0}+\frac{r S^{*}}{K} & \left(\frac{r}{K}+\lambda\right) S^{*} & 0 \\
-\lambda I^{*} & i \omega_{0}-\frac{m I^{*} P^{*}}{\left(a+I^{*}\right)^{2}} & \frac{d}{\alpha} \\
0 & -\frac{\alpha a m P^{*}}{\left(a+I^{*}\right)^{2}} e^{-i \omega_{0} \tau^{(j)}} & i \omega_{0}+d-d e^{-i \omega_{0} \tau^{(j)}}
\end{array}\right) q(0)=\left(\begin{array}{l}
0 \\
0 \\
0
\end{array}\right)
$$

Thus, we can easily obtain

$$
q(0)=(1, \beta, \gamma)^{T}
$$

where

$$
\begin{gathered}
\beta=-\frac{i \omega_{0} K+r S^{*}}{(r+\lambda K) S^{*}}, \\
\gamma=-\frac{\alpha a m P^{*}\left(i \omega_{0} K+r S^{*}\right) e^{-i \omega_{0} \tau^{(j)}}}{(r+\lambda K) S^{*}\left(i \omega_{0}+d-d e^{-i \omega_{0} \tau^{(j)}}\right)} .
\end{gathered}
$$

Similarly, let $q^{*}(s)=D\left(1, \beta^{*}, \gamma^{*}\right)^{T} e^{i s \omega_{0} \tau^{(j)}}$ be the eigenvector of $A^{*}$ corresponding to $-i \omega_{0} \tau^{(j)}$. By the definition of $A^{*}$ and (3.2), (3.3), and (3.4), we can compute

$$
q^{*}(s)=D\left(1, \beta^{*}, \gamma^{*}\right) e^{i s \omega_{0} \tau^{(j)}}=D\left(1, \frac{-i \omega_{0} K+r S^{*}}{\lambda K I^{*}}, \frac{d\left(i \omega_{0} K-r S^{*}\right)}{\alpha\left(-i \omega_{0}+d-d e^{-i \omega_{0} \tau^{(j)}}\right)}\right) e^{i s \omega_{0} \tau^{(j)}}
$$

In order to assure $\left\langle q^{*}(s), q(\theta)\right\rangle=1$, we need to determine the value of $D$. From (3.10), we have

$$
\begin{aligned}
\left\langle q^{*}(s), q(\theta)\right\rangle= & \bar{D}\left(1, \bar{\beta}^{*}, \bar{\gamma}^{*}\right)(1, \beta, \gamma)^{T} \\
& -\int_{-1}^{0} \int_{\xi=0}^{\theta} \bar{D}\left(1, \bar{\beta}^{*}, \bar{\gamma}^{*}\right) e^{-i \omega_{0} \tau^{(j)}(\xi-\theta)} d \eta(\theta)(1, \beta, \gamma)^{T} e^{i \omega_{0} \xi \tau^{(j)}} d \xi \\
= & \bar{D}\left\{1+\beta \bar{\beta}^{*}+\gamma \bar{\gamma}^{*}-\int_{-1}^{0}\left(1, \bar{\beta}^{*}, \bar{\gamma}^{*}\right) \theta e^{i \omega_{0} \theta \tau^{(j)}} d \eta(\theta)(1, \beta, \gamma)^{T}\right\} \\
= & \bar{D}\left\{1+\beta \bar{\beta}^{*}+\gamma \bar{\gamma}^{*}+\tau^{(j)} \bar{\gamma}^{*} \frac{\alpha \beta a m P^{*}}{\left(a+I^{*}\right)^{2}} e^{-i \omega_{0} \tau^{(j)}}\right\} .
\end{aligned}
$$


Thus, we can choose $\bar{D}$ as

$$
\begin{aligned}
& \bar{D}=\frac{1}{1+\beta \bar{\beta}^{*}+\gamma \bar{\gamma}^{*}+\tau^{(j)} \bar{\gamma}^{*}\left(\alpha \beta a m P^{*} /\left(a+I^{*}\right)^{2}\right) e^{-i \omega_{0} \tau^{(j)}}}, \\
& \therefore D=\frac{1}{1+\bar{\beta} \beta^{*}+\bar{\gamma} \gamma^{*}+\tau^{(j)} \gamma^{*}\left(\alpha \beta a m P^{*} /\left(a+I^{*}\right)^{2}\right) e^{i \omega_{0} \tau^{(j)}}} .
\end{aligned}
$$

In the remainder of this section, we use the theory of Hassard et al. [24] to compute the conditions describing center manifold $C_{0}$ at $v=0$. Let $x_{t}$ be the solution of (3.8) when $v=0$. Define

$$
z(t)=\left\langle q^{*}, x_{t}\right\rangle, \quad W(t, \theta)=x_{t}(\theta)-2 \operatorname{Re}\{z(t) q(\theta)\}
$$

On the center manifold $C_{0}$, we have

$$
W(t, \theta)=W(z(t), \bar{z}(t), \theta)
$$

where

$$
W(z, \bar{z}, \theta)=W_{20}(\theta) \frac{z^{2}}{2}+W_{11}(\theta) z \bar{z}+W_{02}(\theta) \frac{\bar{z}^{2}}{2}+W_{30}(\theta) \frac{z^{3}}{6}+\cdots
$$

$z$ and $\bar{z}$ are local coordinates for center manifold $C_{0}$ in the direction of $q^{*}$ and $\bar{q}^{*}$. Note that $W$ is real if $x_{t}$ is real. We only consider real solutions. For solution $x_{t} \in C_{0}$ of (3.8), since $v=0$, we have

$$
\dot{z}(t)=i \omega_{0} \tau^{(j)} z+\bar{q}^{*}(0) f(0, W(z, \bar{z}, 0)+2 \operatorname{Re}\{z q(\theta)\}) \stackrel{\text { def }}{=} i \omega_{0} \tau^{(j)} z+\bar{q}^{*}(0) f_{0}(z, \bar{z}) .
$$

We rewrite this equation as

$$
\dot{z}(t)=i \omega_{0} \tau^{(j)} z(t)+g(z, \bar{z}),
$$

where

$$
g(z, \bar{z})=\bar{q}^{*}(0) f_{0}(z, \bar{z})=g_{20} \frac{z^{2}}{2}+g_{11} z \bar{z}+g_{02} \frac{\bar{z}^{2}}{2}+g_{21} \frac{z^{2} \bar{z}}{2}+\cdots
$$


We have $x_{t}(\theta)=\left(x_{1 t}(\theta), x_{2 t}(\theta), x_{3 t}(\theta)\right)$ and $q(\theta)=(1, \beta, \gamma)^{T} e^{i \theta \omega_{0} \tau^{(j)}}$, so from (3.17) and (3.19) it follows that

$$
\begin{aligned}
x_{t}(\theta)= & W(t, \theta)+2 \operatorname{Re}\{z(t) q(t)\} \\
= & W_{20}(\theta) \frac{z^{2}}{2}+W_{11}(\theta) z \bar{z}+W_{02}(\theta) \frac{\bar{z}^{2}}{2}+(1, \beta, \gamma)^{T} e^{i \omega_{0} \tau^{(j)}} z \\
& +(1, \bar{\beta}, \bar{\gamma})^{T} e^{-i \omega_{0} \tau^{(j)}} \theta \bar{z}+\cdots
\end{aligned}
$$

and then we have

$$
\begin{gathered}
x_{1 t}(0)=z+\bar{z}+W_{20}^{1}(0) \frac{z^{2}}{2}+W_{11}^{(1)}(0) z \bar{z}+W_{02}^{(1)}(0) \frac{\bar{z}^{2}}{2}+\cdots, \\
x_{2 t}(0)=\beta z+\bar{\beta} \bar{z}+W_{20}^{(2)}(0) \frac{z^{2}}{2}+W_{11}^{(2)}(0) z \bar{z}+W_{02}^{(2)}(0) \frac{\bar{z}^{2}}{2}+\cdots \\
x_{3 t}(0)=\gamma z+\bar{\gamma} \bar{z}+W_{20}^{(3)}(0) \frac{z^{2}}{2}+W_{11}^{(3)}(0) z \bar{z}+W_{02}^{(3)}(0) \frac{\bar{z}^{2}}{2}+\cdots \\
x_{1 t}(-1)=z e^{-i \omega_{0} \tau^{(j)}}+\bar{z} e^{i \omega_{0} \tau^{(j)}}+W_{20}^{(1)}(-1) \frac{z^{2}}{2}+W_{11}^{(1)}(-1) z \bar{z}+W_{02}^{(1)}(-1) \frac{\bar{z}^{2}}{2}+\cdots, \\
x_{2 t}(-1)=\beta z e^{-i \omega_{0} \tau^{(j)}}+\bar{\beta} \bar{z} e^{i \omega_{0} \tau^{(j)}}+W_{20}^{(2)}(-1) \frac{z^{2}}{2}+W_{11}^{(2)}(-1) z \bar{z}+W_{02}^{(2)}(-1) \frac{\bar{z}^{2}}{2}+\cdots, \\
x_{3 t}(-1)=\gamma z e^{-i \omega_{0} \tau^{(j)}}+\bar{\gamma} \bar{z} e^{i \omega_{0} \tau^{(j)}}+W_{20}^{(3)}(-1) \frac{z^{2}}{2}+W_{11}^{(3)}(-1) z \bar{z}+W_{02}^{(3)}(-1) \frac{\bar{z}^{2}}{2}+\cdots
\end{gathered}
$$

It follows together with (3.3) that

$$
\begin{aligned}
g(z, \bar{z})= & \bar{q}^{*}(0) f_{0}(z, \bar{z})=\bar{q}^{*}(0) f\left(0, x_{t}\right) \\
= & \tau^{(j)} \bar{D}\left(1, \bar{\beta}^{*}, \bar{\gamma}^{*}\right)\left(\begin{array}{c}
-\frac{r}{K}\left(x_{1 t}^{2}(0)+x_{1 t} x_{2 t}(0)\right)-\lambda x_{1 t}(0) x_{2 t}(0) \\
\lambda x_{1 t}(0) x_{2 t}(0)-\frac{m x_{2 t}(0) x_{3 t}(0)}{a+x_{2 t}(0)} \\
\frac{\alpha m x_{2 t}(-1) x_{3 t}(-1)}{a+x_{2 t}(-1)}
\end{array}\right) \\
= & \frac{z^{2}}{2}\left\{2 \tau^{(j)} D\left[-\left(\frac{r}{K}+\frac{\beta(r+\lambda K)}{K}\right)+\bar{\beta}^{*}\left(\beta \lambda-\frac{m \beta \gamma}{a}\right)+\bar{\gamma}^{*} \frac{m \alpha \beta \gamma e^{-2 i \omega_{0} \tau^{(j)}}}{a}\right]\right\} \\
& +z \bar{z}\left\{2 \tau ^ { ( j ) } \left[D-\left(\frac{r}{K}+\frac{(r+\lambda K)}{K} \operatorname{Re}\{\beta\}\right)+\bar{\beta}^{*}\left(\lambda \operatorname{Re}\{\beta\}-\frac{m}{a} \operatorname{Re}\{\beta \bar{\gamma}\}\right)\right.\right. \\
& \left.\left.+\bar{\gamma}^{*} \frac{m \alpha}{a} \operatorname{Re}\{\beta \bar{\gamma}\}\right]\right\}
\end{aligned}
$$




$$
\begin{gathered}
+\frac{\bar{z}^{2}}{2}\left\{2 \tau^{(j)} D\left[-\left(\frac{r}{K}+\frac{\bar{\beta}(r+\lambda K)}{K}\right)+\bar{\beta}^{*}\left(\bar{\beta} \lambda-\frac{m \bar{\beta} \bar{\gamma}}{a}\right)+\bar{\gamma}^{*} \frac{m \alpha \bar{\beta} \bar{\gamma} e^{2 i \omega_{0} \tau^{(j)}}}{a}\right]\right\} \\
+\frac{z^{2} \bar{z}}{2}\left\{-\frac{\tau^{(j)} D r}{K}\left\{4 W_{11}^{(1)}(0)+2 W_{20}^{(1)}(0)\right\}\right. \\
-\frac{\tau^{(j)} D\left(r+\lambda K-\bar{\beta}^{*} \lambda K\right)}{K}\left\{2 \beta W_{11}^{(1)}(0)+2 W_{11}^{(2)}(0)+\bar{\beta} W_{20}^{(1)}(0)+W_{20}^{(2)}(0)\right\} \\
+\frac{\tau^{(j)} D \bar{\beta}^{*} m}{a}\left\{2 \beta W_{11}^{(3)}(0)+2 \gamma W_{11}^{(2)}(0)+\bar{\beta} W_{20}^{(3)}(0)\right. \\
\left.+\bar{\gamma} W_{20}^{(2)}(0)-\frac{2}{a}\left(\beta^{2} \bar{\gamma}+2 \beta \bar{\beta} \gamma\right)\right\} \\
+\frac{\tau^{(j)} D \bar{\gamma}^{*} m \alpha}{a}\left[2 e^{-i \omega_{0} \tau^{(j)}}\left\{\beta W_{11}^{(3)}(-1)+\gamma W_{11}^{(2)}(-1)\right\}\right. \\
+e^{i \omega_{0} \tau^{(j)}}\left\{\bar{\beta} W_{20}^{(3)}(-1)+\bar{\gamma} W_{20}^{(2)}(-1)\right\} \\
\left.\left.-2 \beta(\beta \bar{\gamma}+2 \bar{\beta} \gamma) e^{-i \omega_{0} \tau^{(j)}}\right]\right\} \ldots
\end{gathered}
$$

Comparing the coefficients with (3.22), we have

$$
\begin{aligned}
g_{20}= & 2 \tau^{(j)} D\left[-\left(\frac{r}{K}+\frac{\beta(r+\lambda K)}{K}\right)+\bar{\beta}^{*}\left(\beta \lambda-\frac{m \beta \gamma}{a}\right)+\bar{\gamma}^{*} \frac{m \alpha \beta \gamma e^{-2 i \omega_{0} \tau^{(j)}}}{a}\right], \\
g_{11}= & 2 \tau^{(j)} D\left[-\left(\frac{r}{K}+\frac{(r+\lambda K)}{K} \operatorname{Re}\{\beta\}\right)+\bar{\beta}^{*}\left(\lambda \operatorname{Re}\{\beta\}-\frac{m}{a} \operatorname{Re}\{\beta \bar{\gamma}\}\right)+\bar{\gamma}^{*} \frac{m \alpha}{a} \operatorname{Re}\{\beta \bar{\gamma}\}\right], \\
g_{02}= & 2 \tau^{(j)} D\left[-\left(\frac{r}{K}+\frac{\bar{\beta}(r+\lambda K)}{K}\right)+\bar{\beta}^{*}\left(\bar{\beta} \lambda-\frac{m \bar{\beta} \bar{\gamma}}{a}\right)+\bar{\gamma}^{*} \frac{m \alpha \bar{\beta} \bar{\gamma} e^{2 i \omega_{0} \tau^{(j)}}}{a}\right], \\
g_{21}= & -\frac{\tau^{(j)} D r}{K}\left\{4 W_{11}^{(1)}(0)+2 W_{20}^{(1)}(0)\right\} \\
& -\frac{\tau^{(j)} D\left(r+\lambda K-\bar{\beta}^{*} \lambda K\right)}{K}\left\{2 \beta W_{11}^{(1)}(0)+2 W_{11}^{(2)}(0)+\bar{\beta} W_{20}^{(1)}(0)+W_{20}^{(2)}(0)\right\} \\
& -\frac{\tau^{(j)} D \bar{\beta}^{*} m}{a}\left\{2 \beta W_{11}^{(3)}(0)+2 \gamma W_{11}^{(2)}(0)+\bar{\beta} W_{20}^{(3)}(0)+\bar{\gamma} W_{20}^{(2)}(0)-\frac{2}{a}\left(\beta^{2} \bar{\gamma}+2 \beta \bar{\beta} \gamma\right)\right\} \\
& +\frac{\tau^{(j)} D \bar{\gamma}^{*} m \alpha}{a}\left[2 e^{-i \omega_{0} \tau^{(j)}}\left\{\beta W_{11}^{(3)}(-1)+\gamma W_{11}^{(2)}(-1)\right\}\right. \\
& \left.+e^{i \omega_{0} \tau^{(j)}}\left\{\bar{\beta} W_{20}^{(3)}(-1)+\bar{\gamma} W_{20}^{(2)}(-1)\right\}-2 \beta(\beta \bar{\gamma}+2 \bar{\beta} \gamma) e^{-i \omega_{0} \tau^{(j)}}\right] .
\end{aligned}
$$

Since there are $W_{20}(\theta)$ and $W_{11}(\theta)$ in $g_{21}$, we still need to compute them. 
From (3.8) and (3.17), we have

$$
\begin{aligned}
\dot{W}=\dot{x}_{t}-\dot{z} q-\dot{z} \bar{q} & = \begin{cases}A W-2 \operatorname{Re}\left\{\bar{q}^{*}(0) f_{0} q(\theta)\right\}, & \theta \in[-1,0), \\
A W-2 \operatorname{Re}\left\{\bar{q}^{*}(0) f_{0} q(\theta)\right\}+f_{0}, & \theta=0,\end{cases} \\
& \stackrel{\text { def }}{=} A W+H(z, \bar{z}, \theta),
\end{aligned}
$$

where

$$
H(z, \bar{z}, \theta)=H_{20}(\theta) \frac{z^{2}}{2}+H_{11}(\theta) z \bar{z}+H_{02}(\theta) \frac{\bar{z}^{2}}{2}+\cdots
$$

Substituting the corresponding series into (3.27) and comparing the coefficients, we obtain

$$
\left(A-2 i \omega_{0} \tau^{j}\right) W_{20}(\theta)=-H_{20}(\theta), \quad A W_{11}(\theta)=-H_{11}(\theta) .
$$

From (3.27), we know that for $\theta \in[-1,0)$,

$$
H(z, \bar{z}, \theta)=-\bar{q}^{*}(0) f_{0} q(\theta)-q^{*}(0) \bar{f}_{0} \bar{q}(\theta)=-g(z, \bar{z}) q(\theta)-\bar{g}(z, \bar{z}) \bar{q}(\theta) .
$$

Comparing the coefficients with (3.28), we get

$$
\begin{aligned}
& H_{20}(\theta)=-g_{20} q(\theta)-\bar{g}_{02} \bar{q}(\theta), \\
& H_{11}(\theta)=-g_{11} q(\theta)-\bar{g}_{11} \bar{q}(\theta) .
\end{aligned}
$$

From (3.29) and (3.31) and the definition of $A$, it follows that

$$
\dot{W}_{20}(\theta)=2 i \omega_{0} \tau^{(j)} W_{20}(\theta)+g_{20} q(\theta)+\bar{g}_{02} \bar{q}(\theta) .
$$

Notice that $q(\theta)=(1, \beta, \gamma)^{T} e^{i \omega_{0} \tau^{(j)} \theta}$, hence

$$
W_{20}(\theta)=\frac{i g_{20}}{\omega_{0} \tau^{(j)}} q(0) e^{i \omega_{0} \tau^{(j)} \theta}+\frac{i \bar{g}_{02}}{3 \omega_{0} \tau^{(j)}} \bar{q}(0) e^{-i \omega_{0} \tau^{(j)} \theta}+E_{1} e^{2 i \omega_{0} \tau^{(j)} \theta},
$$

where $E_{1}=\left(E_{1}^{(1)}, E_{1}^{(2)}, E_{1}^{(3)}\right) \in R^{3}$ is a constant vector. Similarly, from (3.29) and (3.32), we obtain

$$
W_{11}(\theta)=-\frac{i g_{11}}{\omega_{0} \tau^{(j)}} q(0) e^{i \omega_{0} \tau^{(j)} \theta}+\frac{i \bar{g}_{11}}{\omega_{0} \tau^{(j)}} \bar{q}(0) e^{-i \omega_{0} \tau^{(j)} \theta}+E_{2}
$$

where $E_{2}=\left(E_{2}^{(1)}, E_{2}^{(2)}, E_{2}^{(3)}\right) \in R^{3}$ is also a constant vector. 
In what follows, we will seek appropriate $E_{1}$ and $E_{2}$. From the definition of $A$ and (3.29), we obtain

$$
\begin{gathered}
\int_{-1}^{0} d \eta(\theta) W_{20}(\theta)=2 i \omega_{0} \tau^{(j)} W_{20}(0)-H_{20}(0), \\
\int_{-1}^{0} d \eta(\theta) W_{11}(\theta)=-H_{11}(0),
\end{gathered}
$$

where $\eta(\theta)=\eta(0, \theta)$.

By (3.27), we have

$$
\begin{gathered}
H_{20}(0)=-g_{20} q(0)-\bar{g}_{02} \bar{q}(0)+2 \tau^{(j)}\left(\begin{array}{c}
-\frac{r}{K}-\beta\left(\frac{r}{K}+\lambda\right) \\
\beta \lambda-\frac{m \beta \gamma}{a} \\
\frac{m \alpha \beta \gamma}{a} e^{-2 i \omega_{0} \tau^{(j)}}
\end{array}\right) \\
H_{11}(0)=-g_{11} q(0)-\bar{g}_{11} \bar{q}(0)+2 \tau^{(j)}\left(\begin{array}{c}
-\frac{r}{K}-\left(\frac{r}{K}+\lambda\right) \operatorname{Re}\{\beta\} \\
\lambda \operatorname{Re}\{\beta\}-\frac{m}{a} \operatorname{Re}\{\beta \bar{\gamma}\} \\
\frac{m \alpha}{a} \operatorname{Re}\{\beta \bar{\gamma}\}
\end{array}\right) .
\end{gathered}
$$

Substituting (3.34) and (3.38) into (3.36) and noticing that

$$
\begin{gathered}
\left(i \omega_{0} \tau^{(j)} I-\int_{-1}^{0} e^{i \omega_{0} \tau^{(j)} \theta} d \eta(\theta)\right) q(0)=0 \\
\left(-i \omega_{0} \tau^{(j)} I-\int_{-1}^{0} e^{-i \omega_{0} \tau^{(j)} \theta} d \eta(\theta)\right) \bar{q}(0)=0
\end{gathered}
$$

we obtain

$$
\left(2 i \omega_{0} \tau^{(j)} I-\int_{-1}^{0} e^{2 i \omega_{0} \tau^{(j)} \theta} d \eta(\theta)\right) E_{1}=2 \tau^{(j)}\left(\begin{array}{c}
-\frac{r}{K}-\beta\left(\frac{r}{K}+\lambda\right) \\
\beta \lambda-\frac{m \beta \gamma}{a} \\
\frac{m \alpha \beta \gamma}{a} e^{-2 i \omega_{0} \tau^{(j)}}
\end{array}\right)
$$


This leads to

$$
\left(\begin{array}{ccc}
2 i \omega_{0}+\frac{r}{K} S^{*} & \left(\frac{r}{K}+\lambda\right) S^{*} & 0 \\
-\lambda I^{*} & 2 i \omega_{0}-\frac{m I^{*} P^{*}}{\left(a+I^{*}\right)^{2}} & \frac{d}{\alpha} \\
0 & -\frac{a m \alpha P^{*}}{\left(a+I^{*}\right)^{2}} e^{-2 i \omega_{0} \tau^{(j)}} & 2 i \omega_{0}+d-d e^{-2 i \omega_{0} \tau^{(j)}}
\end{array}\right) E_{1}=2\left(\begin{array}{c}
-\frac{r}{K}-\beta\left(\frac{r}{K}+\lambda\right) \\
\beta \lambda-\frac{m \beta \gamma}{a} \\
\frac{m \alpha \beta \gamma}{a} e^{-2 i \omega_{0} \tau^{(j)}}
\end{array}\right)
$$

Solving this system for $E_{1}$, we obtain

$$
\begin{aligned}
& E_{1}^{(1)}=\frac{2}{A}\left|\begin{array}{ccc}
-\frac{r}{K}-\beta\left(\frac{r}{K}+\lambda\right) & \left(\frac{r}{K}+\lambda\right) S^{*} & 0 \\
\beta \lambda-\frac{m \beta \gamma}{a} & 2 i \omega_{0}-\frac{m I^{*} P^{*}}{\left(a+I^{*}\right)^{2}} & \frac{d}{\alpha} \\
\frac{m \alpha \beta \gamma}{a} e^{-2 i \omega_{0} \tau^{(j)}} & -\frac{a m \alpha P^{*}}{\left(a+I^{*}\right)^{2}} e^{-2 i \omega_{0} \tau^{(j)}} & 2 i \omega_{0}+d-d e^{-2 i \omega_{0} \tau^{(j)}}
\end{array}\right| \text {, } \\
& E_{1}^{(2)}=\frac{2}{A}\left|\begin{array}{ccc}
2 i \omega_{0}+\frac{r}{K} S^{*}-\frac{r}{K}-\beta\left(\frac{r}{K}+\lambda\right) & 0 \\
-\lambda I^{*} & \beta \lambda-\frac{m \beta \gamma}{a} & \frac{d}{\alpha} \\
0 & \frac{m \alpha \beta \gamma}{a} e^{-2 i \omega_{0} \tau^{(j)}} & 2 i \omega_{0}+d-d e^{-2 i \omega_{0} \tau^{(j)}}
\end{array}\right|, \\
& E_{1}^{(3)}=\frac{2}{A}\left|\begin{array}{ccc}
2 i \omega_{0}+\frac{r}{K} S^{*} & \left(\frac{r}{K}+\lambda\right) S^{*} & -\frac{r}{K}-\beta\left(\frac{r}{K}+\lambda\right) \\
-\lambda I^{*} & 2 i \omega_{0}-\frac{m I^{*} P^{*}}{\left(a+I^{*}\right)^{2}} & \beta \lambda-\frac{m \beta \gamma}{a} \\
0 & -\frac{a m \alpha P^{*}}{\left(a+I^{*}\right)^{2}} e^{-2 i \omega_{0} \tau^{(j)}} & \frac{m \alpha \beta \gamma}{a} e^{-2 i \omega_{0} \tau^{(j)}}
\end{array}\right|
\end{aligned}
$$

where

$$
A=\left|\begin{array}{ccc}
2 i \omega_{0}+\frac{r}{K} S^{*} & \left(\frac{r}{K}+\lambda\right) S^{*} & 0 \\
-\lambda I^{*} & 2 i \omega_{0}-\frac{m I^{*} P^{*}}{\left(a+I^{*}\right)^{2}} & \frac{d}{\alpha} \\
0 & -\frac{a m \alpha P^{*}}{\left(a+I^{*}\right)^{2}} e^{-2 i \omega_{0} \tau^{(j)}} & 2 i \omega_{0}+d-d e^{-2 i \omega_{0} \tau^{(j)}}
\end{array}\right|
$$


Similarly, substituting (3.35) and (3.39) into (3.37), we get

$$
\left(\begin{array}{ccc}
\frac{r}{K} S^{*} & \left(\frac{r}{K}+\lambda\right) S^{*} & 0 \\
-\lambda I^{*} & -\frac{m I^{*} P^{*}}{\left(a+I^{*}\right)^{2}} & \frac{d}{\alpha} \\
0 & -\frac{a m \alpha P^{*}}{\left(a+I^{*}\right)^{2}} & 0
\end{array}\right) E_{2}=2\left(\begin{array}{c}
-\frac{r}{K}-\left(\frac{r}{K}+\lambda\right) \operatorname{Re}\{\beta\} \\
\lambda \operatorname{Re}\{\beta\}-\frac{m}{a} \operatorname{Re}\{\beta \bar{r}\} \\
\frac{m \alpha}{a} \operatorname{Re}\{\beta \bar{\gamma}\}
\end{array}\right)
$$

and hence

$$
\begin{aligned}
& E_{2}^{(1)}=\frac{2}{B}\left|\begin{array}{ccc}
-\frac{r}{K}-\left(\frac{r}{K}+\lambda\right) \operatorname{Re}\{\beta\} & \left(\frac{r}{K}+\lambda\right) S^{*} & 0 \\
\lambda \operatorname{Re}\{\beta\}-\frac{m}{a} \operatorname{Re}\{\beta \bar{\gamma}\} & -\frac{m I^{*} P^{*}}{\left(a+I^{*}\right)^{2}} & \frac{d}{\alpha} \\
\frac{m \alpha}{a} \operatorname{Re}\{\beta \bar{\gamma}\} & -\frac{a m \alpha P^{*}}{\left(a+I^{*}\right)^{2}} & 0
\end{array}\right|, \\
& E_{2}^{(2)}=\frac{2}{B}\left|\begin{array}{ccc}
\frac{r}{K} S^{*} & -\frac{r}{K}-\left(\frac{r}{K}+\lambda\right) \operatorname{Re}\{\beta\} & 0 \\
-\lambda I^{*} & \lambda \operatorname{Re}\{\beta\}-\frac{m}{a} \operatorname{Re}\{\beta \bar{\gamma}\} & \frac{d}{\alpha} \\
0 & \frac{m \alpha}{a} \operatorname{Re}\{\beta \bar{\gamma}\} & 0
\end{array}\right|, \\
& E_{2}^{(3)}=\frac{2}{B}\left|\begin{array}{ccc}
\frac{r}{K} S^{*} & \left(\frac{r}{K}+\lambda\right) S^{*} & -\frac{r}{K}-\left(\frac{r}{K}+\lambda\right) \operatorname{Re}\{\beta\} \\
-\lambda I^{*} & -\frac{m I^{*} P^{*}}{\left(a+I^{*}\right)^{2}} & \lambda \operatorname{Re}\{\beta\}-\frac{m}{a} \operatorname{Re}\{\beta \bar{\gamma}\} \\
0 & -\frac{a m \alpha P^{*}}{\left(a+I^{*}\right)^{2}} & \frac{m \alpha}{a} \operatorname{Re}\{\beta \bar{\gamma}\}
\end{array}\right|,
\end{aligned}
$$

where

$$
B=\left|\begin{array}{ccc}
\frac{r}{K} S^{*} & \left(\frac{r}{K}+\lambda\right) S^{*} & 0 \\
-\lambda I^{*} & -\frac{m I^{*} P^{*}}{\left(a+I^{*}\right)^{2}} & \frac{d}{\alpha} \\
0 & -\frac{a m \alpha P^{*}}{\left(a+I^{*}\right)^{2}} & 0
\end{array}\right|
$$




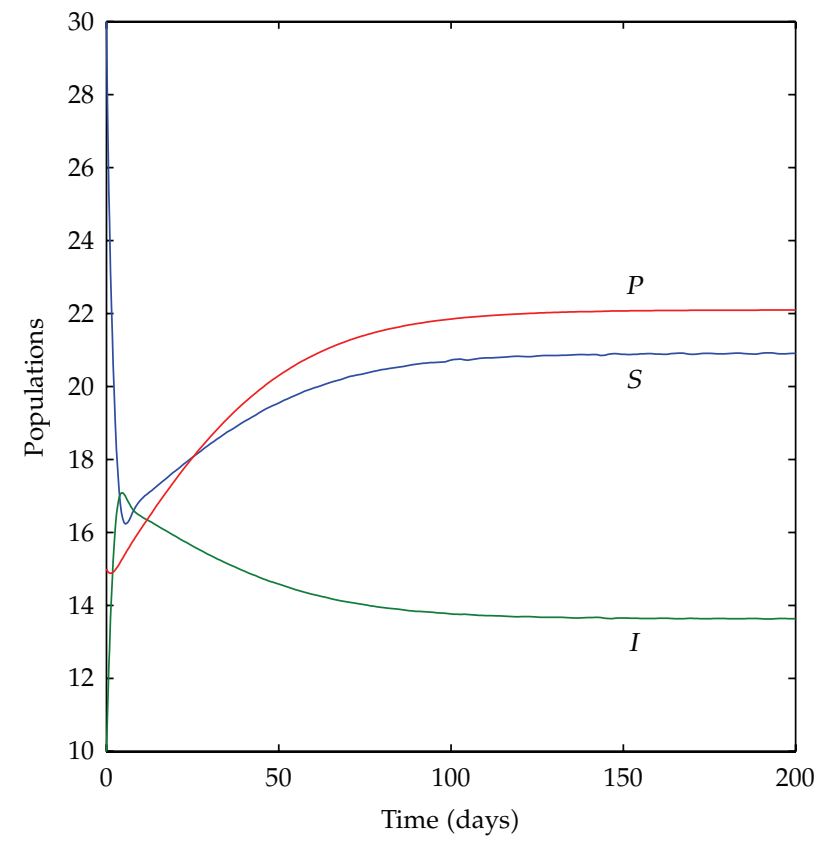

Figure 1: Stable coexistence of prey and predator for $\tau=0$. Parameter values are given in the text.

Thus, we can determine $W_{20}(\theta)$ and $W_{11}(\theta)$ from (3.34) and (3.35). Furthermore, $g_{21}$ in (3.26) can be expressed by the parameters and delay. Thus, we can compute the following values:

$$
\begin{gathered}
c_{1}(0)=\frac{i}{2 \omega_{0} \tau^{(j)}}\left(g_{20} g_{11}-2\left|g_{11}\right|^{2}-\frac{\left|g_{02}\right|^{2}}{3}\right)+\frac{g_{21}}{2}, \\
v_{2}=-\frac{\operatorname{Re}\left\{c_{1}(0)\right\}}{\operatorname{Re}\left\{\xi^{\prime}\left(\tau^{(j)}\right)\right\}}, \\
\beta_{2}=2 \operatorname{Re}\left\{c_{1}(0)\right\}, \\
T_{2}=-\frac{\operatorname{Im}\left\{c_{1}(0)\right\}+\nu_{2} \operatorname{Im}\left\{\xi^{\prime}\left(\tau^{(j)}\right)\right\}}{\omega_{0} \tau^{(j)}},
\end{gathered}
$$

which determine the qualities of bifurcating periodic solution in the center manifold at the critical value $\tau^{(j)}$.

Theorem 3.1. $v_{2}$ determines the direction of the Hopf bifurcation. If $v_{2}>0$, then the Hopf bifurcation is supercritical and the bifurcating periodic solutions exist for $\tau>\tau^{(j)}$. If $\nu_{2}<0$, then the Hopf bifurcation is subcritical and the bifurcating periodic solutions exist for $\tau<\tau^{(j)} . \beta_{2}$ determines the stability of the bifurcating periodic solutions: the bifurcating periodic solutions are stable if $\beta_{2}<0$ and unstable if $\beta_{2}>0 . T_{2}$ determines the period of the bifurcating periodic solutions: the period increase if $T_{2}>0$ and decrease if $T_{2}<0$. 


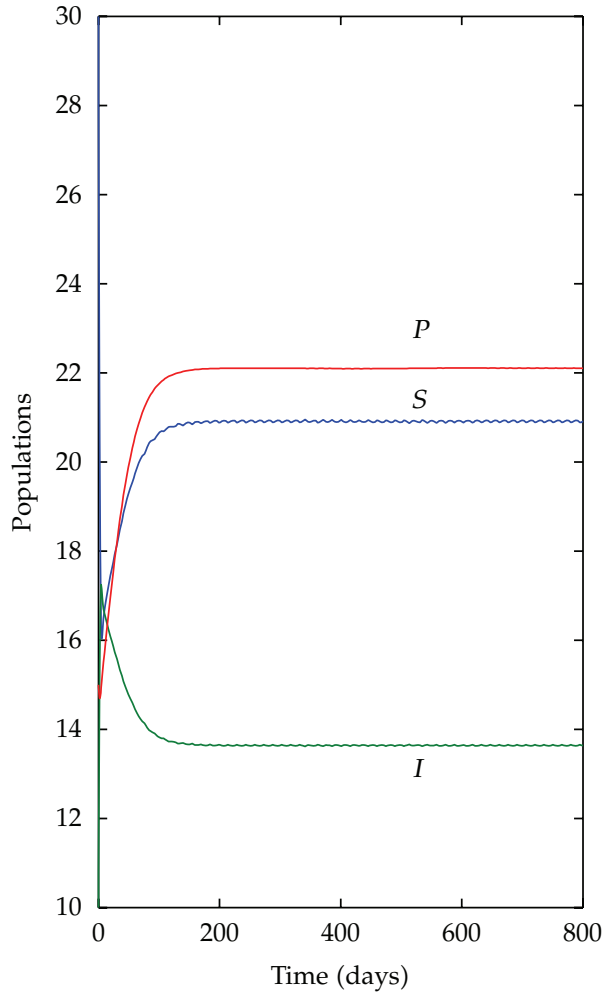

(a)

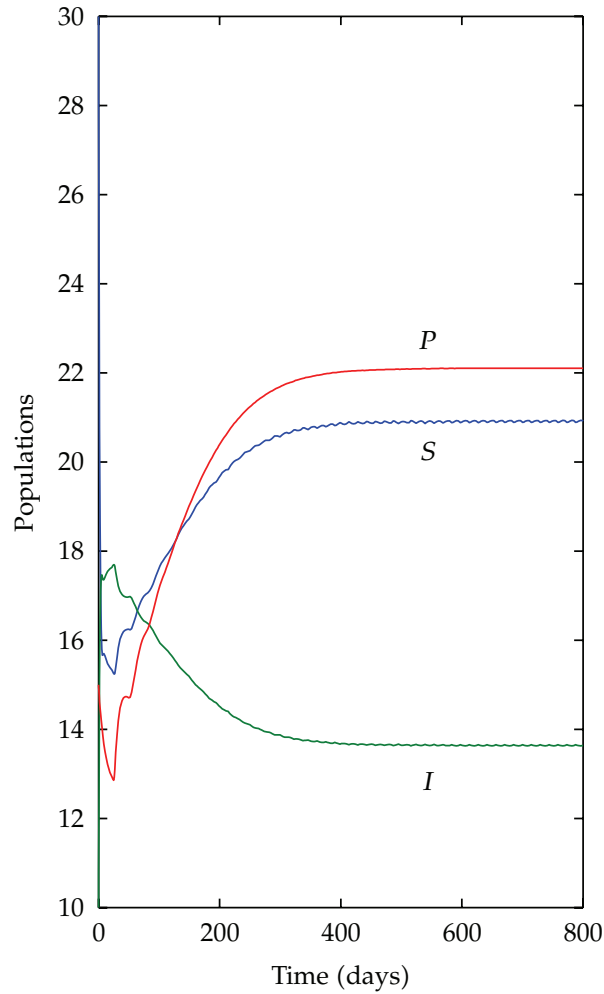

(b)

Figure 2: Time series solutions of the prey and predator populations of the system (1.2): (a) $\tau=1$, (b) $\tau=25$. Parameter values are given in the text. This figure shows that the coexisting equilibrium $E^{*}$ is absolutely stable for all delay.

\section{Numerical Simulations}

In this section, we present some numerical simulations to illustrate the analytical results observed in the previous sections. We consider the following set of parameter values:

$$
r=3, \quad K=40, \quad \lambda=0.03, \quad m=0.45, \quad a=15, \quad \mu=0.28, \quad \alpha=0.42, \quad d=0.09 .
$$

For the above parameter set, the system (1.2) has a unique coexistence equilibrium point $E^{*}=\left(S^{*}, I^{*}, P^{*}\right)=(20.9091,13.6364,22.0992)$. When $\tau \geq 0$, the system (1.2) satisfies all conditions of the Theorem 2.9(i). Consequently, the coexistence equilibrium point $E^{*}$ becomes absolutely stable. Figure 1 shows the behavior of the system (1.2) when $\tau=0$, and Figure 2 depicts the same for $\tau=1$ and $\tau=25$. If we change the value of $m$ from 0.45 to 0.72 in the given parameter set, then conditions of the Theorem 2.9(ii) are satisfied and the system (1.2) becomes conditionally stable around the coexistence equilibrium point $E^{*}$ for $\tau \in\left[0, \tau_{0}\right)$ (see, Figure 3(a)) and unstable for $\tau>\tau_{0}$ (see, Figure 3(b)).

For the given parameter set with $m=0.72$, one can evaluate that $\tau_{0}=2.3187$ and $g^{\prime}\left(h_{k}\right)=0.3312 \neq 0$, so the system (1.2) undergoes a Hopf bifurcation at $E^{*}$ when $\tau=\tau_{0}$ following the condition (iii) of Theorem 2.9. We have constructed a bifurcation diagram (see, Figure 4 ) to observe the dynamics of the system when $\tau$ varies. For this, we have run the 


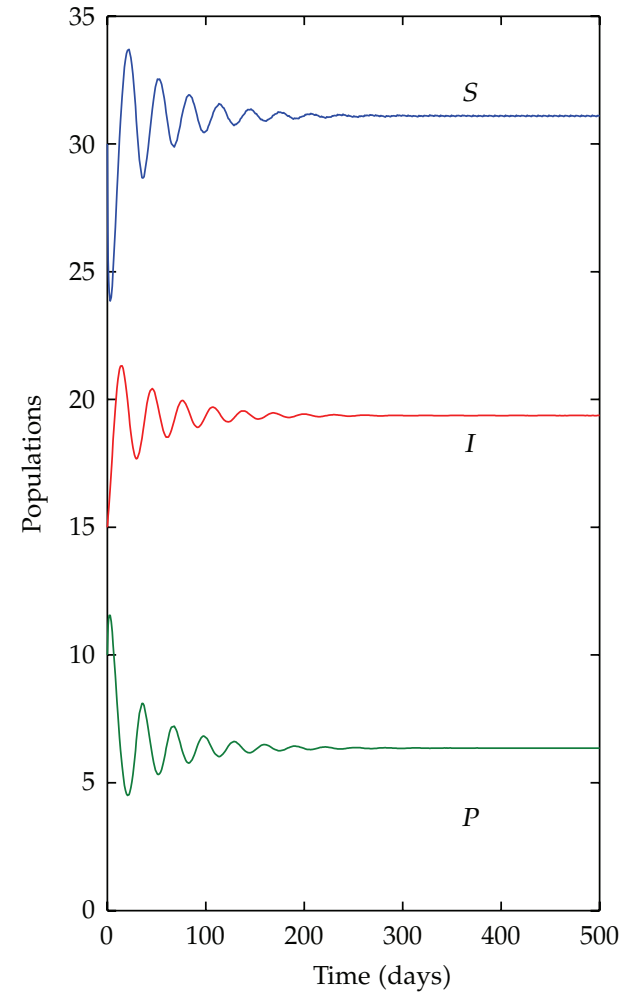

(a)

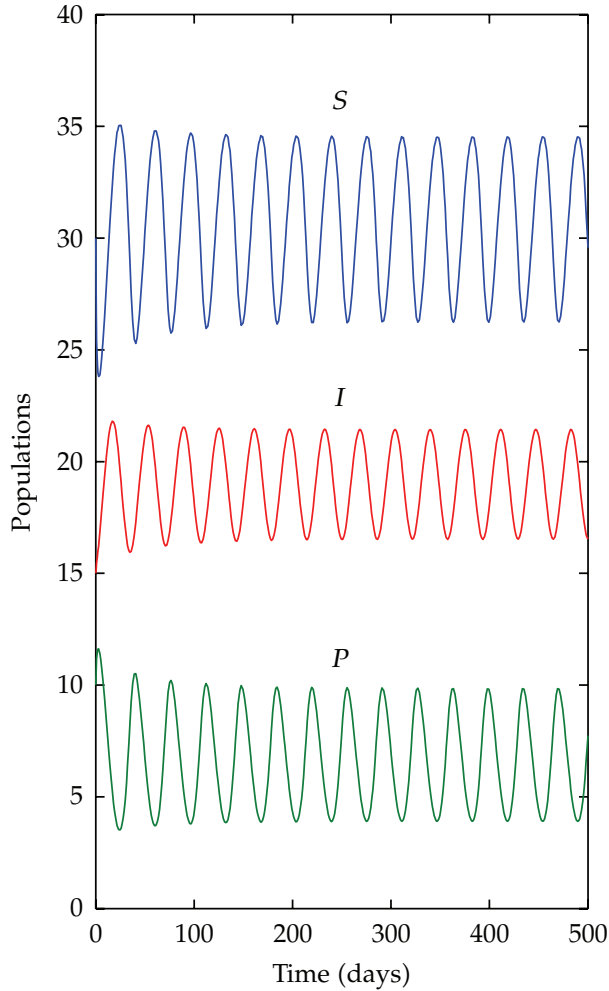

(b)

Figure 3: Behavior of the system (1.2) for different $\tau$ : (a) $\tau=1$, (b) $\tau=3$. All parameters are as in Figure 2 except $m=0.72$. This figure represents the conditional stability of the coexisting equilibrium $E^{*}$.

system (1.2) for 500 time-steps and have plotted the successive maxima and minima of the prey and predator populations with $\tau$ as a variable parameter. This figure shows that the coexisting equilibrium $E^{*}$ is stable if $\tau$ is less than its critical value $\tau_{0}=2.3187$ and unstable if $\tau>\tau_{0}$ and a Hopf bifurcation occurs at $\tau=\tau_{0}$.

Using Theorem 3.1, one can determine the values of $v_{2}, \beta_{2}$ and $T_{2}$. For the given parameter set with $m=0.72$, one can evaluate that $v_{2}=58.4107(>0), \beta_{2}=-1.3298(<0)$, and $T_{2}=1.9364(>0)$. Since $v_{2}>0$ and $\beta_{2}<0$, the Hopf bifurcation is supercritical and the bifurcating periodic solutions exist when $\tau$ crosses $\tau_{0}$ from left to right. Also, the bifurcating periodic solution is stable (as $\beta_{2}<0$ ) and its period increases with $\tau$ (as $T_{2}>0$ ). From the bifurcation diagram (Figure 4), it is clear that when the delay, $\tau$, exceeds the critical value $\tau_{0}$ (= 2.3187 days approximately), the system (2.4) bifurcates from stable focus to stable limit cycle. One can also notice that the amplitude of the oscillations increases with increasing $\tau$.

\section{Summary}

In this paper, we have studied the effects of reproduction delay on an ecoepidemiological system where predator-prey interaction follows Holling Type II response function. We have obtained sufficient conditions on the parameters for which the delay-induced system is asymptotically stable around the positive equilibrium for all values of the delay parameter 

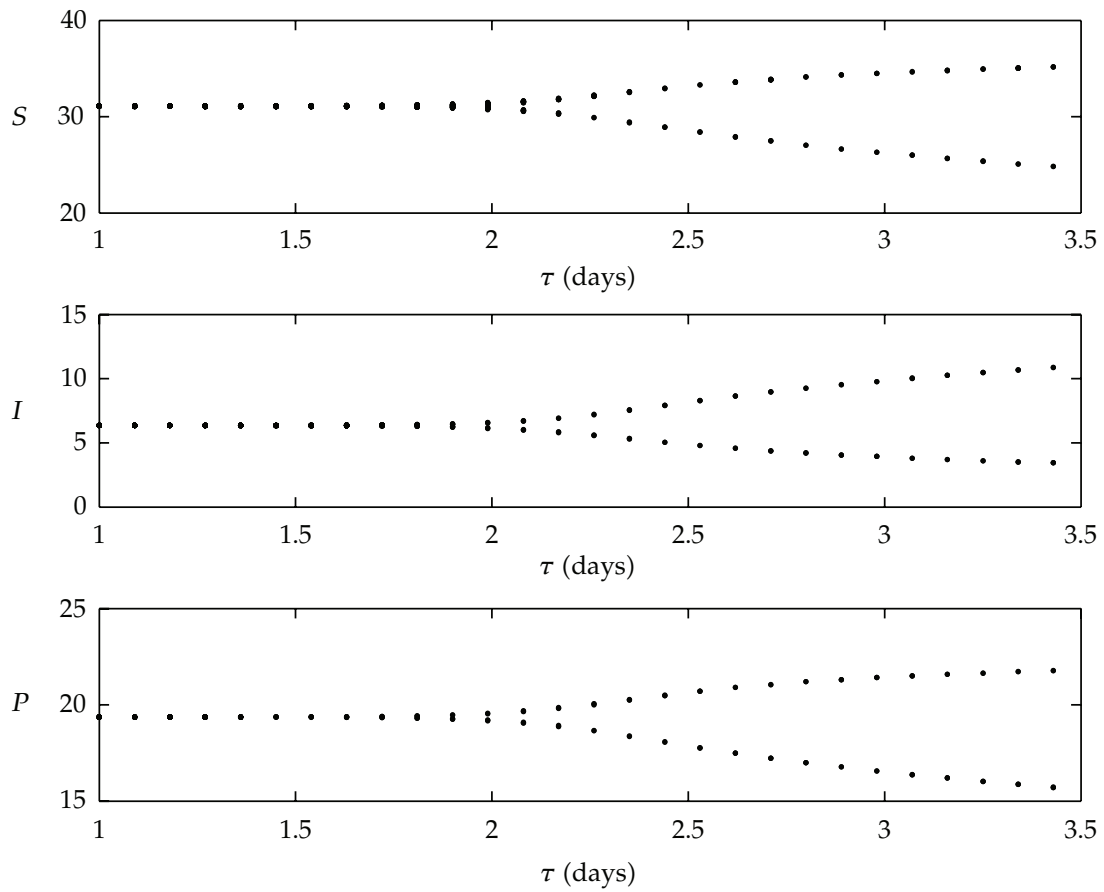

Figure 4: Bifurcation diagram of the susceptible prey, infected prey and predator populations with respect to the delay $\tau$. Parameters are as in Figure 3(b). This figure shows that the coexisting equilibrium $E^{*}$ is stable if $\tau<\tau_{0}=2.3187$ and unstable if $\tau>\tau_{0}$.

and if the conditions are not satisfied, then there exists a critical value of the delay parameter below which the system is stable and above which the system is unstable. By applying the normal form theory and the center manifold theorem, the explicit formulae which determine the stability and direction of the bifurcating periodic solutions have been determined. Our analytical and simulation results show that when $\tau$ passes through the critical value $\tau_{0}$, the coexisting equilibrium $E^{*}$ losses its stability and a Hopf bifurcation occurs, that is, a family of periodic solutions bifurcate from $E^{*}$. Also, the amplitude of oscillations increases with increasing $\tau$. For the considered parameter values, it is observed that the Hopf bifurcation is supercritical and the bifurcating periodic solution is stable. The quantitative level of abundance of system populations depends crucially on the delay parameter if the reproduction period of predator exceeds the critical value $\tau_{0}$.

\section{Acknowledgment}

Research is supported by DST (PURSE seheme), India; no. SR/54/MS: 408/06. The author wishes to thank the anonymous referee for careful reading of the paper.

\section{References}

[1] R. M. Anderson and R. M. May, "The invasion, persistence and spread of infectious diseases within animal and plant communities," Philosophical Transactions of the Royal Society of London B, vol. 314, no. 1167, pp. 533-570, 1986. 
[2] H. I. Freedman, "A model of predator-prey dynamics as modified by the action of a parasite," Mathematical Biosciences, vol. 99, no. 2, pp. 143-155, 1990.

[3] J. Chattopadhyay and N. Bairagi, "Pelicans at risk in Salton sea-an eco-epidemiological model," Ecological Modelling, vol. 136, no. 2-3, pp. 103-112, 2001.

[4] N. Bairagi, P. K. Roy, and J. Chattopadhyay, "Role of infection on the stability of a predator-prey system with several response functions-a comparative study," Journal of Theoretical Biology, vol. 248, no. 1, pp. 10-25, 2007.

[5] N. Bairagi, S. Chaudhuri, and J. Chattopadhyay, "Harvesting as a disease control measure in an ecoepidemiological system-a theoretical study," Mathematical Biosciences, vol. 217, no. 2, pp. 134-144, 2009.

[6] S. R. Hall, M. A. Duffy, and C. E. Cáceres, "Selective predation and productivity jointly drive complex behavior in host-parasite systems," American Naturalist, vol. 165, no. 1, pp. 70-81, 2005.

[7] H. W. Hethcote, W. Wang, L. Han, and Z. Ma, "A predator-prey model with infected prey," Theoretical Population Biology, vol. 66, no. 3, pp. 259-268, 2004.

[8] E. Venturino, "Epidemics in predator-prey models: disease in the predators," IMA Journal of Mathematics Applied in Medicine and Biology, vol. 19, no. 3, pp. 185-205, 2002.

[9] Y. Xiao and L. Chen, "Modeling and analysis of a predator-prey model with disease in the prey," Mathematical Biosciences, vol. 171, no. 1, pp. 59-82, 2001.

[10] C. S. Holling, "Some characteristics of simple types of predation and parasitism," Canadian Entomologist, vol. 91, no. 7, pp. 385-398, 1959.

[11] K. D. Lafferty and A. K. Morris, "Altered behaviour of parasitized killfish increases susceptibility to predation by bird final hosts," Ecology, vol. 77, no. 5, pp. 1390-1397, 1996.

[12] J. C. Holmes and W. M. Bethel, "Modification of intermediate host behavior by parasites," in Behavioral Aspects of Parasite Transmission, E. V. Canning and C. A. Wright, Eds., vol. 51 of Zoological Journal of the Linnean Society, supplement 1, pp. 123-149, 1972.

[13] K. D. Lafferty, "Foraging on prey that are modified by parasites," American Naturalist, vol. 140, no. 5, pp. 854-867, 1992.

[14] S. Ruan, "Absolute stability, conditional stability and bifurcation in Kolmogorov-type predator-prey systems with discrete delays," Quarterly of Applied Mathematics, vol. 59, no. 1, pp. 159-173, 2001

[15] X. Wen and Z. Wang, "The existence of periodic solutions for some models with delay," Nonlinear Analysis: Real World Applications, vol. 3, no. 4, pp. 567-581, 2002.

[16] K. Li and J. Wei, "Stability and Hopf bifurcation analysis of a prey-predator system with two delays," Chaos, Solitons and Fractals, vol. 42, no. 5, pp. 2606-2613, 2009.

[17] H.-Y. Yang and Y.-P. Tian, "Hopf bifurcation in REM algorithm with communication delay," Chaos, Solitons and Fractals, vol. 25, no. 5, pp. 1093-1105, 2005.

[18] Y. Qu and J. Wei, "Bifurcation analysis in a time-delay model for prey-predator growth with stagestructure," Nonlinear Dynamics, vol. 49, no. 1-2, pp. 285-294, 2007.

[19] C. Çelik, "The stability and Hopf bifurcation for a predator-prey system with time delay," Chaos, Solitons and Fractals, vol. 37, no. 1, pp. 87-99, 2008.

[20] B. Grenfell and M. Keeling, Dynamics of Infectious Disease in Theoretical Ecology, R. M. May and R. A. McLean, Eds., 3rd edition, 2007.

[21] F. Brauer, "Absolute stability in delay equations," Journal of Differential Equations, vol. 69, no. 2, pp. 185-191, 1987.

[22] Y. Kuang, Delay Differential Equations with Applications in Population Dynamics, vol. 191 of Mathematics in Science and Engineering, Academic Press, Boston, Mass, USA, 1993.

[23] S. Ruan and J. Wei, "On the zeros of a third degree exponential polynomial with applications to a delayed model for the control of testosterone secretion," IMA Journal of Mathemathics Applied in Medicine and Biology, vol. 18, no. 1, pp. 41-52, 2001.

[24] B. D. Hassard, N. D. Kazarinoff, and Y. H. Wan, Theory and Applications of Hopf Bifurcation, vol. 41 of London Mathematical Society Lecture Note Series, Cambridge University Press, Cambridge, UK, 1981. 


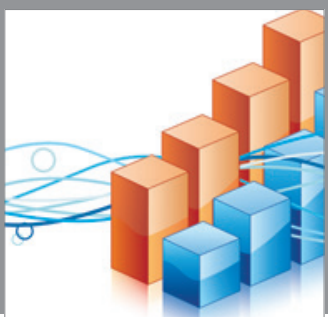

Advances in

Operations Research

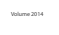

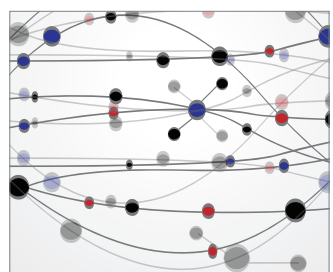

\section{The Scientific} World Journal
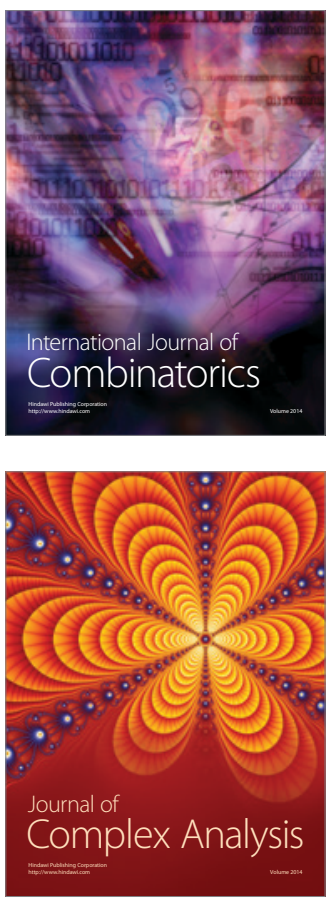

International Journal of

Mathematics and

Mathematical

Sciences
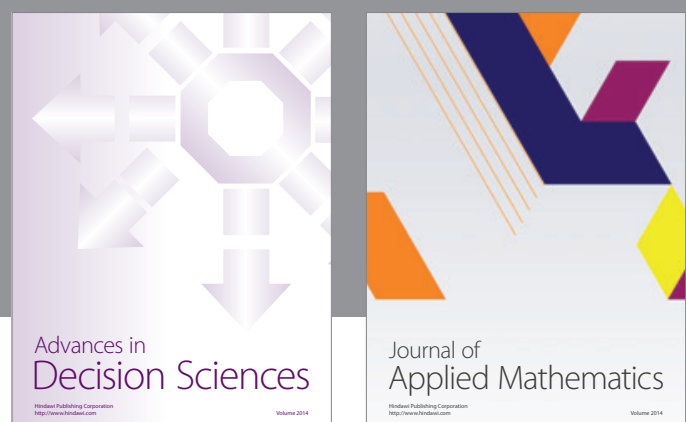

Journal of

Applied Mathematics
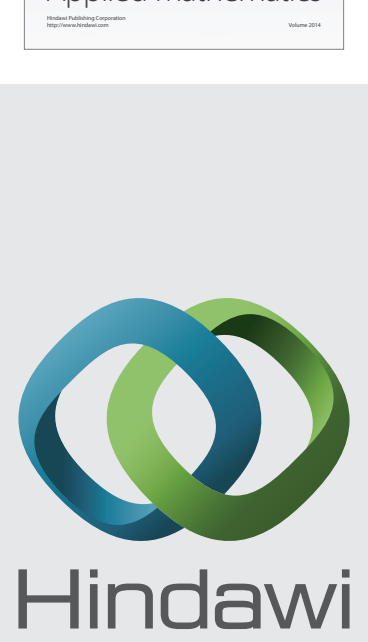

Submit your manuscripts at http://www.hindawi.com
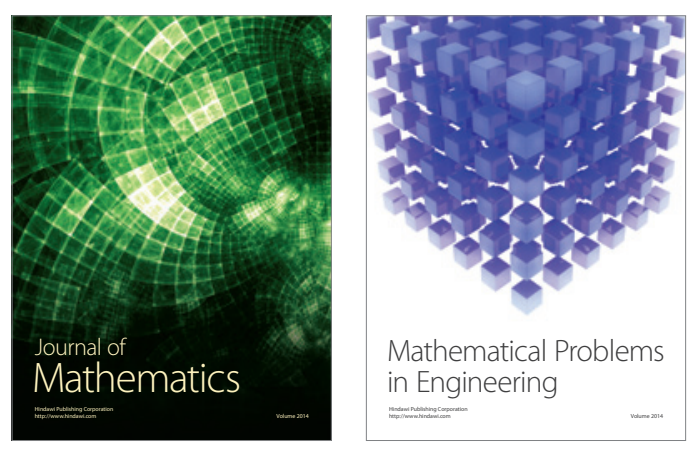

Mathematical Problems in Engineering
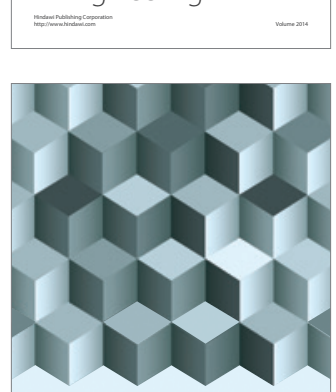

Journal of

Function Spaces
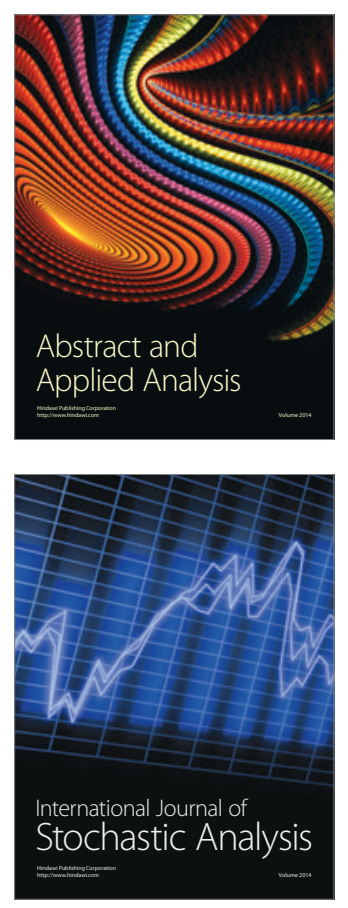

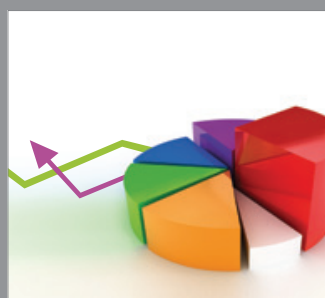

ournal of

Probability and Statistics

Promensencen
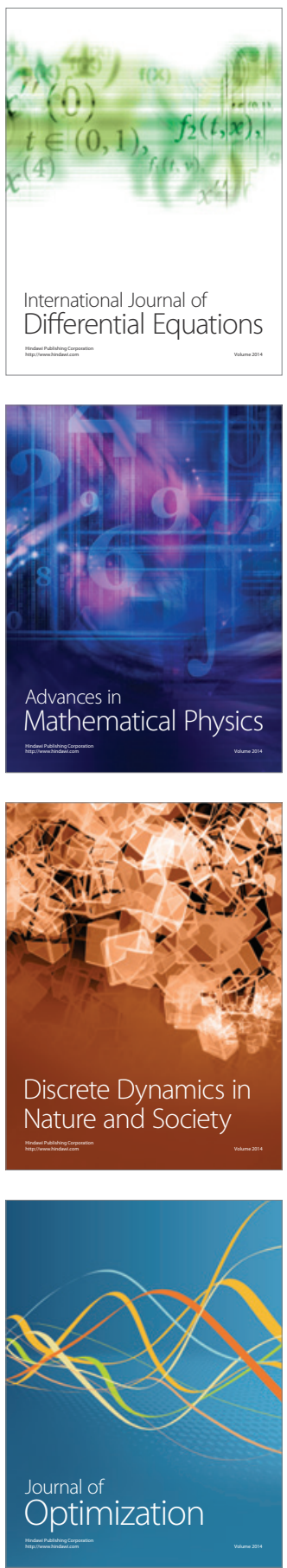